ARTÍCULOS ORIGINALES Rev Chil Salud Pública 2014; Vol 18 (1): 43-51

\section{"ELLOS TAMBIÉN CUENTAN". LA MORTALIDAD DE LOS NIÑOS Y ADOLESCENTES DE 5-19 AÑOS. (ARGENTINA, 1997-2010)}

\author{
"They also count". Mortality among 5-19 year OlD \\ CHILDREN AND ADOLESCENTS (ARGENTINA, 1997-2010)
}

\section{RESUMEN}

Objetivo: El presente trabajo propone analizar la evolución y composición de la mortalidad de la población de 5-19 años en la República Argentina por causas evitables, teniendo en cuenta la atención diferencial que la misma ha recibido en relación a la que tiene lugar en las primeras edades.

Material y método: Se utiliza fundamentalmente información producida por la Dirección de Estadística e Información de Salud (Ministerio de Salud de la Nación, Argentina) y el Instituto Nacional de Estadisticas y Censos, a partir de la cual se calculan tasas especificas de mortalidad por sexo, edad quinquenal y grupos de causas definidos basadas en la combinación de distintas clasificaciones confeccionadas por el Sistema Único de Salud de Brasil y la Organización Mundial de la Salud.

Resultados: Los principales resultados acusan un incremento de los niveles de mortalidad asociados a las causas evitables, lo que deriva, en algunos casos, en el aumento de su participación en el total de defunciones registradas, más acentuado en los varones entre 15 y 19 años. Asimismo, se observa un incremento en los niveles de mortalidad por accidentes de transporte, bomicidios y suicidios.

Conclusiones: No obstante los retrocesos que se detectan en la garantía de los derechos a la salud y a la vida de la población bajo estudio, cabe destacar la incipiente puesta en marcha de acciones estatales destinadas a revertir esta situación.

Palabras clave: mortalidad, causas evitables, niños de 5-9 años, adolescentes. 


\section{ABSTRACT}

Objective: This paper aims to analyze the evolution and breakdown of mortality due to preventable causes in the population of 5-19 year olds in Argentina, given the relatively limited attention it has received compared to children under 5 years old.

Material and Methods: Data produced by the Statistics and Health Information Office (Ministry of Health of the Nation, Argentina) and the National Institute of Statistics and Censuses are used to calculate mortality rates by sex, five-year age group and group of causes, which are constructed from the combination of different classifications generated by the Brazilian Unified Health System and the World Health Organization.

Results: The main results show an increase in the levels of mortality associated with preventable causes. This is related to the increase of their proportion in the total number of deaths, which is more pronounced among 15-19 years old men. Also, there is an increase in mortality levels related to transportation accidents, homicides and suicides.

Conclusions: Despite the setbacks in guaranteeing the rights to bealth and life of the population under study, there are recent state actions that attempt to reverse this situation.

Keywords: mortality, preventable causes, 5-9 year old children, adolescents.

\section{INTRODUCCIÓN}

Las defunciones evitables que tienen lugar en la niñez constituyen un impedimento para el ejercicio de los derechos a la salud y a la vida, al tiempo que reflejan las dificultades que experimentan los niños y sus hogares de pertenencia en el ejercicio de otros derechos sociales, económicos y culturales (1). Por tal motivo, la Convención sobre los Derechos del Niño (CDN) de 1989, primer instrumento jurídicamente vinculante que incorpora toda la gama de derechos humanos (2), precisa en su artículo 24: "Los Estados Partes asegurarán la plena aplicación de este derecho [derecho a la salud] y, en particular, adoptarán las medidas apropiadas para reducir la mortalidad infantil y en la niñez" (3).

Si bien los enunciados de la CDN alcanzan a todas las personas menores de 18 años (artículo 1), es notable que los esfuerzos de los Estados adherentes se hayan centrado en reducir el nivel de defunciones ocurridas en el transcurso de la "primera infancia" (0-4 años). Como contrapartida, en ocasiones, se ha descuidado a los restantes grupos etarios contemplados en la citada convención, al argumentarse que su salud se ve amenazada por "pocas enfermedades que ponen en riesgo sus vidas”.(4) Esta cuestión se expresa en los resultados de diversas investigaciones. Entre ellas, la realizada por Viner y colaboradores en el año 2010, quienes, además de evidenciar la atención diferencial que reciben las personas sobrevivientes a los primeros años de vida en relación a los esfuerzos para reducir la mortalidad, destacan que la mayor parte de las defunciones adolescentes responde a causas evitables, externas en su mayoría, con una creciente participación de los accidentes, la violencia y el suicidio (5).

La República Argentina, país que ratifica la CDN en 1990,(6) no resulta ajena a la problemática planteada. En este sentido, sobre la base de las tasas de mortalidad publicadas por el Centro Latinoamericano y Caribeño de Demografía (CELADE) - División de Población de la Comisión Económica para América Latina y el Caribe (CEPAL),(7) es posible observar que el nivel de mortalidad entre los quinquenios 1990-95 y 2005-2010 desciende al $45 \%, 39 \%, 14 \%, 29 \%$ y $25 \%$, respectivamente, en los varones menores de 1 año y de 1-4, 5-9, 10-14 y 15-19 años; mientras que en las mujeres se reduce el $46 \%, 44 \%, 21 \%, 26 \%$ y $30 \%$, considerando los mismos tramos etarios. 
Las diferencias que registra Argentina en términos de la dinámica de la mortalidad de los niños y adolescentes, suscitan diversos interrogantes respecto de aquellos que consiguen superar las primeras edades: ¿Han resultado suficientes los esfuerzos realizados por el país para reducir la participación de las muertes evitables en este grupo, en función de los compromisos asumidos desde la adhesión a la CDN? ¿Qué causas han logrado reducirse en mayor proporción? ¿Cuáles constituyen un desafío pendiente en materia de políticas públicas para dar continuidad al descenso de la mortalidad?

Con el propósito de buscar una respuesta a estos interrogantes, se propone analizar la evolución y composición de la mortalidad de la población de 5-19 años por causas evitables, de acuerdo a las fuentes de información y los criterios metodológicos precisados en el siguiente apartado.

\section{MATERIAL Y MÉTODO}

Como fuera anticipado, si bien la CDN alcan$\mathrm{za}$ a todas las personas menores de 18 años, el presente trabajo propone un análisis de las tendencias de la mortalidad de la población de 5-19 años. La incorporación del tramo de 18 y 19 años se realiza conforme a la definición de adolescencia de la Organización Mundial de la Salud, la cual refiere a la etapa que transcurre entre los 10 y 19 años de edad (8).

Considerada la anterior aclaración, se calculan y analizan tasas específicas de mortalidad por sexo y grupos quinquenales de edad, a partir de procesamientos efectuados sobre las bases de defunciones producidas por la Dirección de Estadísticas e Información de Salud (DEIS) (Ministerio de Salud de la Nación, Argentina) y las proyecciones de población confeccionadas por el Instituto Nacional de Estadísticas y Censos (INDEC) de Argentina. A fin de evitar los efectos que pudiese tener cualquier evento aleatorio sobre la tendencia que estas describen en el tiempo, dichas tasas se construyen como el resultado de promedios trienales.

A su vez, dado que Argentina no dispone de una clasificación de muertes evitables y no evi- tables para la población comprendida entre 5 y 19 años (en rigor, la DEIS cuenta con una, aplicable solo a los niños menores de 1 año), para su identificación se recurre a la desagregación propuesta en el ámbito del Sistema Único de Salud (SUS) de Brasil, que abarca la población de 5-74 años de edad (9) y que es elaborada sobre la base de las causas de muerte incluidas en la Clasificación Internacional de Enfermedades - Décima Revisión (CIE 10). Aun cuando se reconoce que dicha clasificación no responde enteramente a la realidad nacional que se pretende describir, se la emplea porque constituye la única de la región conformada por los denominados países del Cono Sur (Argentina, entre ellos) que permite identificar causas de muerte susceptibles de ser contrarrestadas en la población de interés (9).

Asimismo, si bien la clasificación del SUS posibilita la desagregación de las muertes evitables en subgrupos definidos según el tipo de medidas que podría haber contribuido a su impedimento (9), con posterioridad a la identificación de dichas muertes, estas se sistematizan de acuerdo a los grupos de causas utilizados en el trabajo de Viner y colaboradores (5), a saber: violencia, suicidio, accidentes de transporte, otras causas externas no intencionales, enfermedades no transmisibles, enfermedades transmisibles y complicaciones maternas (Tabla 1). Esta decisión, basada en la consideración de una mayor capacidad de los subgrupos seleccionados por los citados autores para describir causas de muerte de manera concreta, se apoya, además, en la clasificación de enfermedades publicada por Organización Mundial de la Salud (WHO, por sus siglas en inglés) en 2008, igualmente tenida en cuenta por los mismos (10).

En este sentido, cabe mencionar que la categoría "enfermedades transmisibles" difiere de la empleada en la publicación de referencia (Enfermedades transmisibles y malnutrición) (5), debido a las dificultades que se presentan para detectar las muertes atribuidas a esta causa entre aquellas evitables, tal y como están definidas las causas incluidas en la lista diseñada en el ámbito del SUS.

Por último, se destaca que, no obstante Argentina adhiere a la CDN en 1990 (año a 
partir del cual debería tener lugar el análisis que aquí se propone, a partir del objetivo establecido en el apartado introductorio), el periodo de análisis se restringe a los trienios 1997-99 y 2008-10. Ello obedece, principalmente, a dos motivos. Por una parte, el país comienza a utilizar la CIE 10 (insumo básico para la aplicación de la lista del SUS) recién en 1997. Por otra, 2010 constituye el último año del cual se encuentra disponible la información referida a defunciones al momento de la confección del presente trabajo.

Tabla 1. Causas evitables del SUS de Brasil según grupos de causas utilizados en Viner y colaboradores (5)

\begin{tabular}{ll}
\hline $\begin{array}{l}\text { Grupos de causas según } \\
\text { Viner y colaboradores }\end{array}$ & Causas incluidas consideradas evitables según el SUS de Brasi \\
Violencia & Agresiones; Intervenciones legales y operaciones de guerra \\
Suicidio & Lesiones autoprovocadas intencionalmente \\
Accidentes de transporte & Accidentes de transporte
\end{tabular}

Quemaduras; Ahogamiento y sumersión accidentales; Exposición al humo, al fuego y a las llamas; Envenenamiento (intoxicación) accidental por exposición a sustancias nocivas; Accidentes ocurridos en pacientes durante la prestación de cuidados médicos o quirúrgicos, reacción anormal en pacientes o complicación tardía causadas por procedimientos quirúrgicos y otros procedimientos médicos sin mención del accidente al tiempo del procedimiento; Incidentes adversos durante los

Otras causas externas no intencionales

Enfermedades no transmisibles

Enfermedades transmisibles

Complicaciones maternas diagnósticos o terapias asociadas al uso de dispositivos médicos; Exposición a fuerzas mecánicas inanimadas; Exposición a fuerzas mecánicas animadas; Otros riesgos accidentales de la respiración; Exposición a corriente eléctrica, a radiación y a temperatura y presión extremas del ambiente; Contacto con una fuente de calor y con sustancias calientes; Contacto con animales y plantas venenosas; Exposición a fuerzas de la naturaleza; Exposición accidental a otros factores no especificados; Efectos adversos de drogas, medicamentos o sustancias biológicas usadas con finalidad terapéutica; Eventos (o actos) cuya intención es indeterminada.

Neoplasia maligna del labio, melanoma maligno de la piel u otras neoplasias malignas de la piel; Neoplasia maligna del hígado y de las vías biliares intra-hepáticas; Neoplasia maligna del estómago; Neoplasia maligna del colon, de la unión retosigmoidea, del recto, del ano o del canal anal; Neoplasia maligna de la boca, de la faringe y de la laringe; Neoplasia maligna del esófago; Neoplasia maligna de la tráquea, de los bronquios y de los pulmones; Neoplasia maligna de la mama; Neoplasia maligna del cuello del útero; Neoplasia maligna de los testículos: Neoplasia maligna de la glándula tiroides; Dolencia de Hodgkin; Leucemia linfoide; Leucemia mieloide; Tirotoxicosis, hipertiroidismo y deficiencias de iodo; Diabetes mellitus; Obesidad; Psicosis alcohólica y otros trastornos del alcohol; Epilepsia y estado de mal epiléptico; Dolencias hipertensivas, excepto hipertensión secundaria; Dolencias isquémicas del corazón; Ateroesclerosis; Insuficiencia cardíaca; Dolencias cerebrovasculares; Dolencias crónicas de las vías aéreas inferiores y edema pulmonar, no especificado de otra forma; Úlcera gástrica, duodenal; Úlcera péptica de localización no especificada y gastroyenunal; Apendicitis aguda; Dolencias pulmonares debidas a agentes externos; Hernias, íleo paralítico y obstrucción intestinal sin hernia; Trastornos de la vesícula biliar y de las vías biliares; Insuficiencia renal crónica.

Tuberculosis miliar; Tétanos obstétrico; Tétano; Difteria; Coqueluche; Poliomielitis aguda; Sarampión; Rubeola; Hepatitis aguda B; Meningitis por Hemofilos; Tuberculosis respiratoria, con confirmación bacteriológica e histológica; Tuberculosis de las vías respiratorias, sin confirmación bacteriológica o histológica; Tuberculosis de otros órganos; Secuelas de tuberculosis; Dolencias infecciosas intestinales; Dolencias por el Virus de la Inmunodeficiencia Humana (HIV), Hepatitis virales (excepto Hepatitis aguda B); Sífilis, gonorreas y otras dolencias sexualmente transmisibles; Dolencias inflamatorias de los órganos pélvicos femeninos; Fiebre reumática aguda y dolencias reumáticas crónicas del corazón; Infecciones respiratorias, inclusive neumonía e influenza; Infecciones de la piel o del tejido subcutáneo, excepto síndrome de la piel escaldada estafilocócica del recién nacido e impétigo; Infección del tracto urinario de localización no especificada; Otras dolencias de notificación compulsiva; Otras infecciones.

Complicaciones del embarazo, parto o puerperio, excepto las vinculadas a la asistencia materna por otras complicaciones ligadas predominantemente al embarazo

Fuente: Elaboración propia basada en Viner y colaboradores (2011), WHO (2008) y lista de causas de muertes evitables utilizada por el SUS de Brasil. 


\section{RESULTADOS}

En términos generales, se puede señalar que, durante los trienios analizados, más del $50 \%$ de las muertes contabilizadas entre los niños $\mathrm{y}$ adolescentes argentinos corresponden a causas susceptibles de ser evitadas mediante la utilización de recursos disponibles. Esta participación (que refleja, fundamentalmente, un grave impedimento para el ejercicio de los derechos a la salud y a la vida), aumenta conforme se incrementa la edad de las personas. Al mismo tiempo, resulta más acentuada entre los varones, encontrándose, además, que la brecha entre sexos se amplifica a medida que se pasa de la niñez a la adolescencia y transcurre esta última (Tabla 2).

Con respecto a las variaciones que experimenta el indicador en cuestión en el periodo abordado, aunque no existe un patrón evidente, también constituyen una muestra de la falta de progresos en materia de derechos humanos. En este sentido, mientras que entre los niños de 5-9 años la tendencia muestra un descenso, en el caso de las niñas del mismo tramo etario acusa un aumento. Así también, independientemente del sexo, registra un incremento entre los adolescentes de 15-19 años. Por su parte, entre aquellos de 10-14 años, la proporción de defunciones evitables se mantiene constante (Tabla 2).

De manera complementaria, la tasa de mortalidad evitable en los grupos estudiados es superior en los varones en todos los casos, a la vez que las diferencias entre sexos se agudizan conforme se asciende en la edad de las personas, sobre todo si se tienen en cuenta los adolescentes varones de 15-19 años (Tabla 3).

En lo referente a la evolución de las tasas exhibidas entre los trienios estudiados, estas logran reducirse en el grupo de 5-14 años. Por el contrario, entre los adolescentes de 1519 años, el nivel de la mortalidad asociado a las causas evitables sufre un incremento en el tiempo, traducido en $6,2 \%$ y $3,6 \%$ en varones y mujeres, en ese orden, comportamiento que guarda estrecha relación con el incremento de las participación de dichas muertes en el total de defunciones registradas (Tabla 3 ).
Tabla 2. Argentina. Participación de las causas evitables en el nivel de la mortalidad de la población de 5-19 años por sexo y grupos de edad (en porcentajes). Trienios 1997-99/2008-10

\begin{tabular}{ccccc}
\hline \multicolumn{5}{c}{$\begin{array}{r}\text { Proporción de muertes evitables en el total de } \\
\text { muertes registradas (en \%) }\end{array}$} \\
\hline $\begin{array}{c}\text { Grupo } \\
\text { de edad }\end{array}$ & \multicolumn{2}{c}{ Varones } & \multicolumn{2}{c}{ Mujeres } \\
& $1997-99$ & $2008-10$ & $1997-99$ & $2008-10$ \\
$5-9$ & 60 & 56 & 49 & 52 \\
$10-14$ & 66 & 65 & 57 & 57 \\
$15-19$ & 77 & 82 & 65 & 69 \\
\hline
\end{tabular}

Aclaración: Los fallecidos de sexo ignorado son distribuidos de manera proporcional entre varones y mujeres.

Fuente: Elaboración propia basada en datos producidos por la DEIS y el INDEC a partir de la clasificación de muertes evitables confeccionada en el ámbito del SUS de Brasil.

Tabla 3. Argentina. Tasas de mortalidad por causas evitables de la población de 5-19 años por sexo y grupos de edad (por 100.000 habitantes). Trienios 1997-99/2008-10

Tasa de mortalidad por causas evitables

\begin{tabular}{ccccc}
\hline $\begin{array}{c}\text { Grupo } \\
\text { de edad }\end{array}$ & \multicolumn{2}{c}{ Varones } & \multicolumn{2}{c}{ Mujeres } \\
& $1997-99$ & $2008-10$ & $1997-99$ & $2008-10$ \\
$5-9$ & 19,9 & 15,1 & 11,9 & 11,5 \\
$10-14$ & 24,4 & 21,9 & 14,8 & 14,4 \\
$15-19$ & 83,9 & 89,1 & 30,8 & 31,9 \\
\hline
\end{tabular}

Aclaración: Los fallecidos de sexo ignorado son distribuidos de manera proporcional entre varones y mujeres.

Fuente: Elaboración propia basada en datos producidos por la DEIS y el INDEC a partir de la clasificación de muertes evitables confeccionada en el ámbito del SUS de Brasil.

Respecto de las causas de las defunciones, una primera lectura del Gráfico 1 permite inferir que, independientemente del sexo y el grupo de edad, aquellas catalogadas como externas no intencionales (excluidos los accidentes de transporte) constituyen las más frecuentes entre las muertes evitables. Esta participación en la estructura de la mortalidad por causas permanece inalterable más allá 
de registrarse un descenso de la tasa asociada a dichas muertes entre el inicio y el final del período en estudio en casi la totalidad de los grupos analizados.

No obstante lo anterior, y aunque no está precisado en el gráfico, si se realiza un análisis detallado al interior del mencionado grupo de causas, ninguno de los niveles de defunciones vinculados con las causas específicas que lo componen -lideradas por los eventos de intención no determinada, seguidos de la exposición accidental a factores no especificados, el ahogamiento y la sumersión accidentales, la exposición a fuerzas mecánicas inanimadas y la exposición a corriente eléctrica, radiación, temperatura y presión extrema-, logra superar a los accidentes de transporte, los cuales, si bien de manera aparente configuran la segunda causa de muerte en la estructura, en realidad, la lideran en la mayoría de los casos (con excepción de las mujeres de 5-9 y de 10-14 años, para las cuales ocupan, respectivamente, el cuarto y quinto lugar en el ranking). Esta posición permanece constante a lo largo del periodo examinado al registrarse un incremento en el nivel de defunciones ligadas a esta causa, salvo entre los varones de 5-14 años y las mujeres de 10-14 años, donde experimenta un ascenso (ver Gráfico 1).

No menos alarmante resulta el aumento de las muertes por causas violentas, que se acentúa conforme aumenta la edad, especialmente entre los varones de 15-19 años. Una realidad similar se observa en el caso de las defunciones debidas a suicidios, cuya participación adquiere mayor importancia en el último trienio analizado, como consecuencia del incremento de las tasas vinculadas (ver Gráfico 1).

Por su parte, el peso relativo de las enfermedades transmisibles y no transmisibles, que generalmente denota grupos de causas que ocupan entre el tercer y quinto lugar del ranking, disminuye a medida que se incrementa la edad de las personas. Asimismo, tiende a registrar una reducción en el tiempo casi en la mayor parte de los grupos investigados (con excepción de algunos casos, en que se ve aumentado como consecuencia del incremento de la tasa asociada) (ver Gráfico 1).
Finalmente, con relación a las muertes atribuidas a complicaciones maternas, aunque no destacan por su representatividad al interior de la estructura de la mortalidad femenina, exhiben un incremento en la tasa vinculada en el caso de las adolescentes de 15-19 años. Esto último impacta en el incremento de su participación en el total de muertes evitables, igualmente inaceptable desde el marco de los derechos humanos (ver Gráfico 1).

\section{DISCUSIÓN}

Mediante el desarrollo del presente trabajo se ha buscado dar respuesta a diversos interrogantes. El primero de ellos apunta a inferir, con base en la evidencia, en qué medida Argentina ha realizado los esfuerzos suficientes para reducir la participación de las muertes evitables entre los niños y adolescentes de 5-19 años, considerando las implicancias que dichas muertes tienen en el contexto de los derechos humanos. En este sentido, los resultados revelan que la tasa de mortalidad asociada a dichas muertes ha conseguido reducirse en el caso de las personas cuyas edades están comprendidas entre 5 y 14 años. Sin embargo, y como contrapartida, dicha tasa acusa un incremento entre los adolescentes de 15-19 años, lo que deriva en el aumento de la participación de las muertes asociadas en la estructura de defunciones del grupo etario y, por lo tanto, responde de manera negativa a la pregunta formulada.

Las conclusiones anteriores coinciden con las plasmadas en trabajos previos confeccionados a nivel local. Entre ellos, el de Serfaty y colaboradoras (11), quienes destacan el considerable aumento que experimenta, a escala nacional, el nivel de la mortalidad masculina en el periodo 1991-2000. Asimismo, se encuentran en consonancia con los aportes efectuados desde otros países para la región (5).

Por su parte, el segundo y tercer interrogante planteados refieren a cuáles son las causas que han logrado reducirse en mayor proporción dentro del grupo, y cuáles representan un desafío pendiente en materia de políticas públicas. En general, los resultados 
Gráfico 1. Argentina. Estructura y nivel de la mortalidad evitable de la población de 5-19 años por causas seleccionadas según sexo y grupo de edad. Periodo 1997-2009
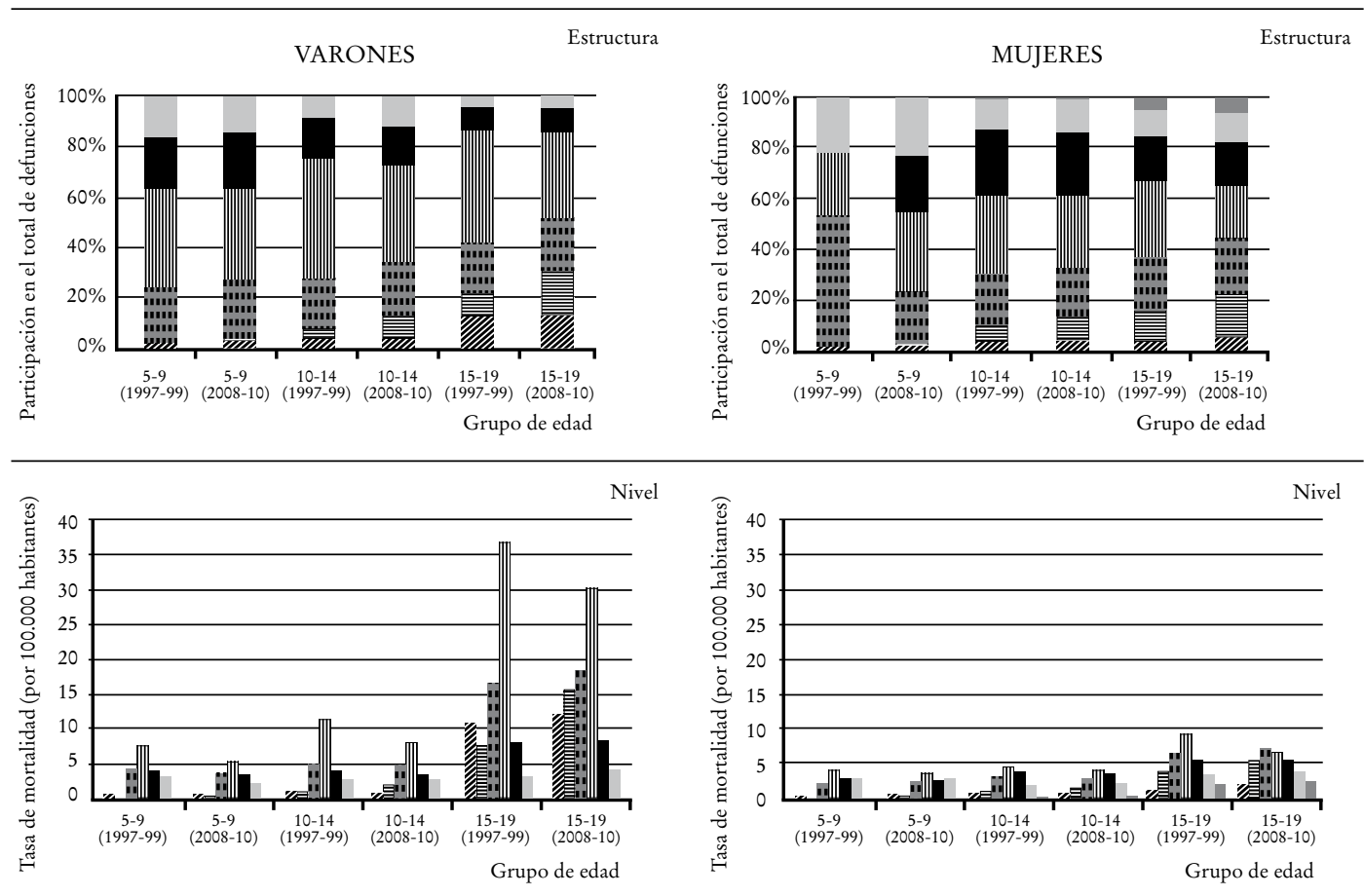

\begin{tabular}{|c|c|c|c|}
\hline WIIll/, Violencia & [IIIII Accidentes de transporte & Enfermedades no transmisibles & Complicaciones maternas \\
\hline 衰 Suicidio & 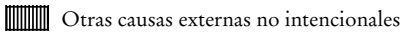 & Enfermedades transmisibles & \\
\hline
\end{tabular}

Aclaraciones: 1. Las causas incluidas dentro de cada categoría se encuentran detalladas en el apartado metodológico. 2. Las tasas de mortalidad se encuentran expresadas por 100.000 habitantes. 3. Se excluyen los fallecidos de sexo ignorado.

Fuente: Elaboración propia basada en datos producidos por la DEIS y el INDEC a partir de la clasificación de muertes evitables confeccionada en el ámbito del SUS de Brasil y la propuesta en OMS (2008).

señalan un descenso del nivel de defunciones originadas en enfermedades transmisibles y no transmisibles. No obstante este progreso, más allá de registrar un descenso entre los años estudiados, las causas externas no intencionales continúan ocupando los primeros lugares dentro del ranking de causas de mortalidad, independientemente del sexo y el grupo de edad, al asociarse al $40 \%$ o más de las defunciones que se producen entre las edades analizadas. Ello se encuentra en consonancia con las reflexiones consignadas por Waisman (12) quien, además, agrega que cualquier esfuerzo para reducir la mortalidad al interior del tramo etario debe comprender necesariamente la reducción de los accidentes de transporte, los cuales se constituyen como los más representativos dentro del citado grupo de causas, fundamentalmente entre los varones de 15-19 años.

Asimismo, y de modo alarmante, se registra un incremento de las tasas de mortalidad ligadas a los hechos de violencia (homicidios y suicidios), notablemente más pronunciados al aumentar la edad de la población analizada y con mayor frecuencia entre los adolescentes varones. Esta realidad es igualmente observada para el total del país por Serfaty 
y colaboradoras $(11,13)$ y Yunes y Zuvarew. (14) Adicionalmente, concuerda con la situación detectada a escala regional por estos últimos, en función de un análisis puntual de la tendencia de las defunciones asociadas a este tipo de causas que los mismos realizan, sobre la base de datos correspondientes a la década de 1980 y mediados de la siguiente, provenientes del Sistema de Información Técnica de la Organización Panamericana de la Salud (14). Cabe considerar, también, las coincidencias que encuentran con la investigación llevada a cabo por Viner y colaboradores (5).

A pesar de las conclusiones que se desprenden de esta investigación, debe destacarse la puesta en marcha del Programa SUMAR desde el Ministerio de Salud de la Nación en el año 2012 (15). Con adaptaciones a nivel local, dicho programa constituye una ampliación del denominado Plan Nacer iniciado en 2005 (vinculado específicamente con la atención de la salud de la población materno-infantil), que incorpora, además, a los niños y adolescentes de 6-19 años, a fin de promover el acceso y ejercicio del derecho a la salud de manera equitativa. De este modo, se plantean medidas que contemplan desde controles pediátricos en centros de salud estatales hasta charlas informativas acerca de la prevención de accidentes, la importancia de la salud sexual y reproductiva y el tratamiento de las adicciones.

De manera complementaria, la producción y difusión de datos estadísticos oficiales, un insumo clave para la toma de decisiones en materia de políticas públicas, ha acompañado la iniciativa comentada, al comenzar a desagregarse la información referida al grupo de 5-19 años por edades simples o tramos quinquenales. Simultáneamente, se ha empezado a hacer énfasis en el análisis de aquellas defunciones atribuidas a causas de creciente preocupación, tales como las externas en conjunto, los accidentes de transporte e, incluso, las que involucran las complicaciones maternas (16-20).

Dada la incipiente implementación del Programa SUMAR, no resulta factible, por el momento, realizar un examen sobre el alcance de las acciones comprendidas en dicho programa. Es esperable, entonces, que su evaluación, a futuro, evidencie avances hacia la plena garantía de los derechos de los niños y adolescentes, al tiempo que aporte al desarrollo de nuevas medidas (o rediseño de las vigentes) que tiendan a realizar tales derechos con igualdad de oportunidades en cada uno de los escenarios en que niños y adolescentes desarrollan sus existencias.

\section{AGRADECIMIENTOS}

Se agradece a Carola Bertone, integrante del CIECS (CONICET-UNC), por las sugerencias brindadas durante la ejecución del presente trabajo.

\section{REFERENCIAS}

1. Santillán Pizarro M, Rojas Cabrera E. La reducción de la mortalidad infantil en Argentina, Brasil y Uruguay: entre intenciones, logros y desafíos. Población y Salud en Mesoamérica [en línea]. 2011 enero [consultado 23.01.2011]; 8(2): 1-21. Disponible en: http://www.redalyc.org/articulo. oa $? \mathrm{id}=44615611003$

2. UNICEF [en línea]. Convención sobre los Derechos del Niño: Introducción [consultado 15.03.2009]. Disponible en: http://www. unicef.org/spanish/crc/.

3. Naciones Unidas. Convención sobre los Derechos del Niño. Nueva York: UN; 1989.

4. Maddaleno M, Morello P, Enfante Espíndola F. Salud y desarrollo de adolescentes y jóvenes en Latinoamérica y el Caribe: desafíos para la próxima década. Salud Publica Méx [en línea]. 2003 [consultado 12.04.2012]; 45(1): 132-9. Disponible en: http://www.scielo.org.mx/pdf/spm/ v45s1/15454.pdf

5. Viner R, Coffey C, Colin M, Bloem P, Costello A, Santelli J, Patton G. 50-year mortality trends in children and young people: a study of 50 low-income, middle-income, and high-income countries Lancet. 2011 Apr 2; 377 (9772): 1162-74. doi: 10.1016/ S0140-6736(11)60106-2. 
6. Naciones Unidas. Status of the Convention on the Rights of the Child at 7-12-2012. Naciones Unidas.

7. CEPAL, CELADE. Boletín Demográfico 74 [en línea]. Santiago, Chile, 2004 [consultado 15.04.2012]. Disponible en: http:// www.eclac.cl/publicaciones/xml/3/19673/ bol-dem74.pdf

8. Organización Mundial de la Salud. La salud de los jóvenes: un desafío para la sociedad. Informe de un Grupo de Estudio de la OMS acerca de los jóvenes y la "Salud para Todos en el Año 2000" [en línea]. Serie Informes Técnicos 731. Ginebra: OMS; 1986 [consultado 7.12.2012]. Disponible en: http:// whqlibdoc.who.int/trs/WHO_TRS_731_ spa.pdf

9. Banco de Dados do Sistema Único de Saúde. Mortalidade. CID-10. Lista de Tabulação de Causas Evitáveis de 5 a 74 anos [en línea]. Brasil: Ministerio da Saúde. [consultado el 10.04.2012]. Disponible en: http://tabnet.datasus.gov.br/cgi/sim/obtcid10evit5a74.htm

10. World Health Organization. The global burden of disease: 2004 update [en línea]. Suiza, 2008 [consultado 10.04.2012]. Disponible en: http://www.who.int/healthinfo/global_ burden_disease/GBD_report_2004update_ full.pdf

11. Serfaty E, Foglia V, Masaútis A, Negri G. Mortalidad por causas violentas en adolescentes y jóvenes de Argentina, 1991-2000. Vertex. 2003; 14(2): 40-8.

12. Waisman I. Los pediatras argentinos y las lesiones no intencionales en la infancia. $A r-$ chivos argentinos de pediatría. 2002; 100(4): 275-7.

13. Serfaty E, Andrade J, Foglia V, Masaútis A, Negri G. Suicidio en adolescentes y jóvenes de 10-24 años. Argentina, 1991-2000. Revista APSA Sinopsis. 2006; 22(42): 16-21.

14. Yunes J, Zubarew T. Mortalidad por causas violentas en adolescentes y jóvenes: un desafío para la Región de las Américas. Rev Bras Epidemiol. 1999; 2(3): 102-71.

15. Argentina. Ministerio de Salud de la Nación. Programa SUMAR [en línea] Buenos Aires: Ministerio de Salud [consultado 21.02.2013]. Disponible en: http://www.msal.gov.ar/sumar/
16. Argentina. Ministerio de Salud de la Nación. Dirección de Estadística e Información. Mortalidad materna según variables seleccionadas. Argentina. Año 1998-2007. Buenos Aires: DEIS; 2009.

17. Argentina. Ministerio de Salud de la Nación. Dirección de Estadística e Información. Indicadores seleccionados de salud para la población de 10 a 19 años. Argentina. Año 2008. Buenos Aires: DEIS; 2010.

18. Argentina. Ministerio de Salud de la Nación. Dirección de Estadística e Información. Indicadores seleccionados de salud para la población de 10 a 19 años. Argentina. Año 2009. Buenos Aires: DEIS; 2011.

19. Argentina. Ministerio de Salud de la Nación. Dirección de Estadística e Información Defunciones por causas externas. Argentina. Años 1997-2009. Buenos Aires: DEIS; 2011.

20. Argentina. Ministerio de Salud de la Nación. Dirección de Estadística e Información. Indicadores seleccionados de salud para la población de 10 a 19 años. Argentina. Año 2010. Buenos Aires: DEIS; 2012. 